\title{
Harmonious Representation of PDF's reflecting Large Deviations
}

\author{
Toshihico Arimitsu ${ }^{a}$ and Naoko Arimitsu ${ }^{b}$ \\ ${ }^{a}$ Institute of Physics, University of Tsukuba, Ibaraki 305-8571, Japan \\ ${ }^{\mathrm{b}}$ Graduate School of EIS, Yokohama Nat'l. University, Yokohama 240-8501, Japan
}

\begin{abstract}
The framework of multifractal analysis (MFA) is distilled to the most sophisticated one. Within this transparent framework, it is shown that the harmonious representation of MFA utilizing two distinct Tsallis distribution functions, one for the tail part of probability density function (PDF) and the other for its center part, explains the recently observed PDF's of turbulence in the highest accuracy superior to the analyses based on other models such as the log-normal model and the $p$ model.
\end{abstract}

Key words: multifractal analysis, turbulence, large wings, fat tails, probability distribution function

PACS: 47.27.-i, 47.53.+n, 47.52.+j, 05.90.+m

\section{Introduction}

The multifractal analysis (MFA) is a unified self-consistent approach dealing with those systems exhibiting large deviations. It has been proposed by the present authors $[1,2,3,4,5,6,7,8,9]$ in order to analyze various probability density functions (PDF's) observed in the system of fully developed turbulence. The PDF's, given for variables normalized by their own standard deviations, represent a characteristic large wings (or fat tails) due to the intermittency specific to turbulence. Since the pioneering work by Kolmogorov [10], there appeared so many attractive attempts to analyze the large wings, e.g., the lognormal model $[11,12,13]$, the $p$ model $[14,15]$ etc., but no one was satisfactory. The MFA is constructed with the help of the Tsallis-type distribution function [16] that provides an extremum of the extensive Rényi entropy [17] or the

Email address: arimitsu@cm.ph.tsukuba.ac.jp (Toshihico Arimitsu). 
non-extensive Tsallis entropy [16,18] under appropriate constraints [3]. After a rather preliminary investigation of the $p$ model [1], we developed further to derive the analytical expression for the scaling exponents of velocity structure function [2,3], and to determine the PDF's of the velocity fluctuations [3,4,5], of the velocity derivative [6] and of the fluid particle accelerations [7].

In this paper, we will show that a harmonious representation of PDF's by means of two distinct Tsallis-type distributions provides us with a description for experimentally or simulationally observed PDF's in the highest accuracy compared with other multifractal model such as the log-normal model $[11,12,13]$ and the $p$ model $[14,15]$. In order to perform a transparent comparison, the framework of MFA is rephrased in its most sophisticated fashion in which the tail part of PDF, giving the probabilities of events larger than its standard deviation, is written down once the multifractal spectrum for the spatial distribution of singularities is specified (see (1) below), whereas the center part, giving the probabilities of events smaller than its standard deviation, is assumed to be analyzed by the Tsallis-type PDF for the variable itself $[8,9]$ (see (2) below). The various PDF's extracted out from the DNS conducted by Gotoh et al. [19,20], and the PDF of fluid particle accelerations observed by Bodenschatz et al. in their Lagrangian measurement of particle accelerations $[21,22,23]$ will be analyzed with the help of the multifractal spectrums for the log-normal, the $p$ model and the harmonious representation.

MFA of turbulence rests on the scale invariance of the Navier-Stokes equation for high Reynolds number, and on the assumption that the singularities due to the invariance distribute themselves, multifractally, in physical space. Let us consider the fluctuation $\delta x_{n}=\left|x\left(\bullet+\ell_{n}\right)-x(\bullet)\right|$ of a physical quantity $x$ at the $n$th multifractal step satisfying the scaling law $\left|x_{n}\right| \equiv\left|\delta x_{n} / \delta x_{0}\right|=\delta_{n}^{\alpha \phi / 3}$ with $\delta_{n}=\ell_{n} / \ell_{0}=\delta^{-n}(n=0,1,2, \cdots)$. We call $n$ the multifractal depth which can be real number in the analysis of experimental data. In the following in this paper, we will put $\delta=2$ that is consistent with the energy cascade model. At each step of the cascade, say at the $n$th step, eddies break up into two pieces producing the energy cascade with the energy-transfer rate $\epsilon_{n}$ that represents the rate of transfer of energy per unit mass from eddies with diameter $\ell_{n}$ to those with $\ell_{n+1}$. Then, we see that the derivative $\left|x^{\prime}\right|=\lim _{n \rightarrow \infty} x_{n}^{\prime}$ with the $n$th difference $x_{n}^{\prime}=\delta x_{n} / \ell_{n}$ for the characteristic length $\ell_{n}$ diverges for $\alpha<3 / \phi[24]$. The velocity fluctuation $\delta u_{n}$ is given for $\phi=1$, and the pressure fluctuation $\delta p_{n}$ is for $\phi=2$. The velocity derivative and the fluid particle acceleration are defined, respectively, by $\left|u^{\prime}\right|=\lim _{n \rightarrow \infty} u_{n}^{\prime}$ and $|\overrightarrow{\mathrm{a}}|=\lim _{n \rightarrow \infty} \mathrm{a}_{n}$ with the $n$th velocity difference $u_{n}^{\prime}=\delta u_{n} / \ell_{n}$ and the $n$th pressure difference (the $n$th acceleration) $\mathrm{a}_{n}=\delta p_{n} / \ell_{n}$. Note that the fluid particle acceleration $\vec{a}$ is given by $\vec{a}=\partial \vec{u} / \partial t+(\vec{u} \cdot \vec{\nabla}) \vec{u}$. For the energy transfer rate, $\phi=3$ since it has the scaling relation $\epsilon_{n} / \epsilon_{0}=\delta_{n}{ }^{\alpha-1}$.

The real quantity $\alpha$ is introduced in the scale transformation $[25,26] \vec{x} \rightarrow \vec{x}^{\prime}=$ 
$\lambda \vec{x}, \quad \vec{u} \rightarrow \vec{u}^{\prime}=\lambda^{\alpha / 3} \vec{u}, t \rightarrow t^{\prime}=\lambda^{1-\alpha / 3} t, p \rightarrow p^{\prime}=\lambda^{2 \alpha / 3} p$ that leaves the Navier-Stokes equation $\partial \vec{u} / \partial t+(\vec{u} \cdot \vec{\nabla}) \vec{u}=-\vec{\nabla} p+\nu \nabla^{2} \vec{u}$ of incompressible fluid invariant when the Reynolds number $\operatorname{Re}=\delta u_{\text {in }} \ell_{\text {in }} / \nu$ is large. Here, $\nu$ is the kinematic viscosity, and $p=\check{p} / \rho$ with the thermodynamical pressure $\check{p}$ and the mass density $\rho . \delta u_{\text {in }}$ and $\ell_{\text {in }}$ represent, respectively, the rotating velocity and the diameter of the largest eddies in turbulence. The largest size of eddies is, for example, about the order of mesh size of a grid, inserted in a laminar flow, that produces turbulence downstream.

\section{General framework}

MFA starts with an assignment of the probability, to find a singularity specified by the strength $\alpha$ within the range $\alpha \sim \alpha+d \alpha$, in the form $P^{(n)}(\alpha) d \alpha=$ $\left(\left|f^{\prime \prime}\left(\alpha_{0}\right)\right|\left|\ln \delta_{n}\right| / 2 \pi\right)^{1 / 2} \delta_{n}^{1-f(\alpha)} d \alpha[15,3]$. Here, $f(\alpha)$ represents an appropriate multifractal spectrum defined in the range $\alpha_{\min } \leq \alpha \leq \alpha_{\max }$. Note that $f(\alpha)$ does not dependent on $n$ because of the scale invariance. The multifractal spectrum is related to the mass exponent $\tau(\bar{q})$, defined by $\left\langle\left(\epsilon_{n} / \epsilon\right)^{\bar{q}}\right\rangle=a_{3 \bar{q}} \delta_{n}^{-\tau(\bar{q})+1-\bar{q}}$ with $a_{3 \bar{q}}=\left(\left|f^{\prime \prime}\left(\alpha_{0}\right)\right| /\left|f^{\prime \prime}\left(\alpha_{\bar{q}}\right)\right|\right)^{1 / 2}$, through the Legendre transformation [15]: $f(\alpha)=\alpha \bar{q}+\tau(\bar{q})$ with $\alpha=\alpha_{\bar{q}}=-d \tau(\bar{q}) / d \bar{q}$ and $\bar{q}=d f(\alpha) / d \alpha$. The average $\langle\cdots\rangle$ is taken with $P^{(n)}(\alpha)$, and $\alpha_{0}=\alpha_{\bar{q}=0}=\langle\alpha\rangle$.

The PDF $\Pi_{\phi}^{(n)}\left(x_{n}\right)$, normalized as $\int d x_{n} \Pi_{\phi}^{(n)}\left(x_{n}\right)=1$, is assumed to consists of two parts, i.e., $\Pi_{\phi}^{(n)}\left(x_{n}\right)=\Pi_{\phi, S}^{(n)}\left(x_{n}\right)+\Delta \Pi_{\phi}^{(n)}\left(x_{n}\right)$ where the first term is related to $P^{(n)}(\alpha)$ through $\Pi_{\phi, S}^{(n)}\left(\left|x_{n}\right|\right) d\left|x_{n}\right|=\left[\left(1-2 \gamma_{\phi, 0}^{(n)}\right) / 2\right] P^{(n)}(\alpha) d \alpha$ with the transformation of the variables $\left|x_{n}\right|=\delta_{n}^{\alpha \phi / 3}$, and the second term is responsible to the contributions coming from the dissipative term in the NavierStokes equation violating the invariance under the scale transformation given above. Here, we introduced $\gamma_{\phi, m}^{(n)}$ by $2 \gamma_{\phi, m}^{(n)}=\int d x_{n}\left|x_{n}\right|^{m} \Delta \Pi_{\phi}^{(n)}\left(x_{n}\right)$. Note that we are dealing with the symmetrized part of PDF's by assuming that the large deviation stemmed from the singular first term contributes to symmetric part of PDF's. We have the $m$ th order structure function for $x_{n}$ in the form $\left\langle\left\langle\left|x_{n}\right|^{m}\right\rangle\right\rangle_{\phi} \equiv \int d x_{n}\left|x_{n}\right|^{m} \Pi_{\phi}^{(n)}\left(x_{n}\right)=2 \gamma_{\phi, m}^{(n)}+\left(1-2 \gamma_{\phi, 0}^{(n)}\right) a_{\phi m} \delta_{n}^{\zeta_{\phi m}}$ with the corresponding scaling exponents $\zeta_{3 \bar{q}}=1-\tau(\bar{q})$. For $\phi=1, \zeta_{\phi m}$ reduces to the scaling exponents $\zeta_{m}$ of the $m$ th order velocity structure function.

Let us introduce the PDF $\hat{\Pi}_{\phi}^{(n)}\left(\xi_{n}\right)$, to be compared with observed data, defined by $\hat{\Pi}_{\phi}^{(n)}\left(\xi_{n}\right) d \xi_{n}=\Pi_{\phi}^{(n)}\left(x_{n}\right) d x_{n}$ or by $\hat{\Pi}_{\phi}^{(n)}\left(\xi_{n}\right) d \xi_{n}=\Pi_{\phi}^{(n)}\left(x_{n}^{\prime}\right) d x_{n}^{\prime}$ for the variable $\xi_{n}=x_{n} /\left\langle\left\langle x_{n}^{2}\right\rangle\right\rangle^{1 / 2}=x_{n}^{\prime} /\left\langle\left\langle\left(x_{n}^{\prime}\right)^{2}\right\rangle\right\rangle^{1 / 2}$ both of the fluctuation $x_{n}$ and of the derivative $x_{n}^{\prime}$ normalized by their own standard deviations. For values of the variable $\left|\xi_{n}\right|$ larger than the order of its standard deviation, $\xi_{n}^{*} \leq\left|\xi_{n}\right| \leq \xi_{n}^{\max }$ 
(equivalently, $\alpha_{\min } \leq|\alpha| \leq \alpha^{*}$ ), the $\mathrm{PDF}$ is given by $[8,9]$

$$
\hat{\Pi}_{\phi}^{(n)}\left(\left|\xi_{n}\right|\right) d\left|\xi_{n}\right|=\Pi_{\phi, \mathrm{S}}^{(n)}\left(\left|x_{n}\right|\right) d\left|x_{n}\right|=\left[\left(1-2 \gamma_{\phi, 0}^{(n)}\right) / 2\right] P^{(n)}(\alpha) d \alpha
$$

where $\xi_{n}^{\max }=\bar{\xi}_{n} \delta_{n}^{\phi \alpha_{\min } / 3-\zeta_{2 \phi} / 2}$ with $\bar{\xi}_{n}=\left[2 \gamma_{\phi, 2}^{(n)} \delta_{n}^{-\zeta_{2 \phi}}+\left(1-2 \gamma_{\phi, 0}^{(n)}\right) a_{2 \phi}\right]^{-1 / 2}$. Note that $\xi_{n}^{*} \sim 1$ as can be seen below when we analyze experiments. This tail part represents the large deviations, and manifests itself the multifractal distribution of the singularities due to the scale invariance of the Navier-Stokes equation when its dissipative term can be neglected. The entropy index $q$ should be unique once a turbulent system with a certain Reynolds number is specified. For smaller values of the variable, $\left|\xi_{n}\right| \leq \xi_{n}^{*}$ (equivalently, $\alpha^{*} \leq|\alpha|$ ), we assume that the PDF has the Tsallis-type structure with a new entropy index $q^{\prime}[8,9]$

$$
\begin{aligned}
& \hat{\Pi}_{\phi}^{(n)}\left(\xi_{n}\right) d \xi_{n}=\left[\Pi_{\phi, \mathrm{S}}^{(n)}\left(x_{n}\right)+\Delta \Pi_{\phi}^{(n)}\left(x_{n}\right)\right] d x_{n} \\
& =\bar{\Pi}_{\phi}^{(n)}\left\{1-\left(1-q^{\prime}\right)\left(\phi+3 f^{\prime}\left(\alpha^{*}\right)\right)\left[\left(\xi_{n} / \xi_{n}^{*}\right)^{2}-1\right] / 2 \phi\right\}^{1 /\left(1-q^{\prime}\right)} d \xi_{n}
\end{aligned}
$$

where $\bar{\Pi}_{\phi}^{(n)}=3\left(1-2 \gamma_{\phi, 0}^{(n)}\right)\left|f^{\prime \prime}\left(\alpha_{0}\right)\right|^{1 / 2} / 2 \phi \bar{\xi}_{n}\left(2 \pi\left|\ln \delta_{n}\right|\right)^{1 / 2}$. This center part is responsible to smaller fluctuations of the variable, compared with its standard deviation, stemmed from the dissipative term violating the scale invariance. The entropy index $q^{\prime}$ can be dependent on the distance of two measuring points.

The two parts of the PDF, (1) and (2), are connected at $\xi_{n}^{*}=\bar{\xi}_{n} \delta_{n}{ }^{\phi \alpha^{*} / 3-\zeta_{2 \phi} / 2}$ under the conditions that they have the common value, $\bar{\Pi}_{\phi}^{(n)}$, and the common $\log$-slope, $-\left(\phi+3 f^{\prime}\left(\alpha^{*}\right)\right) / \phi \xi_{n}^{*}$ there. The value $\alpha^{*}$ is the smaller solution of $\zeta_{2 \phi} / 2-\phi \alpha / 3+1-f(\alpha)=0$. The point $\xi_{n}^{*}$ has the characteristics that the dependence of $\hat{\Pi}_{\phi}^{(n)}\left(\xi_{n}^{*}\right)$ on $n$ is minimum for large $n$ (see Fig. 4 in [4]). With the help of (1) and (2), we obtain $\Delta \Pi_{\phi}^{(n)}\left(x_{n}\right)$, and have the analytical formula $2 \gamma_{\phi, m}^{(n)}=\left(K_{\phi, m}^{(n)}-L_{\phi, m}^{(n)}\right) /\left(1+K_{\phi, 0}^{(n)}-L_{\phi, 0}^{(n)}\right)$ where

$$
\begin{aligned}
K_{\phi, m}^{(n)}= & \left(3 \delta_{n}^{(m+1) \phi \alpha^{*} / 3-\zeta_{2 \phi} / 2} / \phi\right) \sqrt{\left|f^{\prime \prime}\left(\alpha_{0}\right)\right| / 2 \pi\left|\ln \delta_{n}\right|} \\
& \times \int_{0}^{1} d z|z|^{m}\left[1-\left(1-q^{\prime}\right)\left(\phi+3 f^{\prime}\left(\alpha^{*}\right)\right)\left(z^{2}-1\right) / 2 \phi\right]^{1 /\left(1-q^{\prime}\right)} \\
L_{\phi, m}^{(n)}= & \delta_{n} \sqrt{\left|f^{\prime \prime}\left(\alpha_{0}\right)\right|\left|\ln \delta_{n}\right| / 2 \pi} \int_{\alpha^{*}}^{\alpha_{\max }} d \alpha \delta_{n}^{m \alpha \phi / 3-f(\alpha)}
\end{aligned}
$$

For later convenience, we introduce here the quantity $\xi_{n, 0}=\bar{\xi}_{n} \delta_{n}^{\phi \alpha_{0} / 3-\zeta_{2 \phi} / 2}$. 
The PDF's $\hat{\Pi}^{(n)}\left(\xi_{n}\right)$ of the velocity fluctuations and of the velocity derivatives are give by the common formula $\hat{\Pi}^{(n)}\left(\xi_{n}\right)=\hat{\Pi}_{\phi=1}^{(n)}\left(\xi_{n}\right)$ for the normalized variable $\xi_{n}=\delta u_{n} /\left\langle\left\langle\left(\delta u_{n}\right)^{2}\right\rangle\right\rangle^{1 / 2}$, whereas the PDF's $\hat{\Lambda}^{(n)}\left(\omega_{n}\right)$ of the pressure fluctuations and of the fluid particle accelerations are given by the common formula $\hat{\Lambda}^{(n)}\left(\omega_{n}\right)=\hat{\Pi}_{\phi=2}^{(n)}\left(\omega_{n}\right)$ for the normalized variable $\omega_{n}=\delta p_{n} /\left\langle\left\langle\left(\delta p_{n}\right)^{2}\right\rangle\right\rangle^{1 / 2}$.

\section{Log-normal model}

In the log-normal model $[11,12,13]$, one consider the ratio $\epsilon_{n} / \epsilon_{n-1}(n=1,2, \cdots)$ as independent stochastic variables, and apply for $n \gg 1$ the central limit theorem to the summation of their logarithms, $\left(n \sigma^{2}\right)^{-1 / 2} \sum_{j=1}^{n} \ln \left(\epsilon_{j} / \epsilon_{j-1}\right)=$ $\left(n \sigma^{2}\right)^{-1 / 2} \ln \left(\epsilon_{n} / \epsilon\right)=\sqrt{n} \sigma^{-1}(1-\alpha) \ln \delta$, to have the Gaussian distribution function $P^{(n)}(\alpha)=\left(n / 2 \pi \sigma^{2}\right)^{1 / 2} \mathrm{e}^{-n\left(\alpha-\alpha_{0}\right)^{2} / 2 \sigma^{2}}$ for the range $-\infty<\alpha<\infty$. Here, we used the scaling relation between $\epsilon_{n}$ and $\alpha$. Then, we have the multifractal spectrum and the mass exponent in the forms $f(\alpha)=1-\left(\alpha-\alpha_{0}\right)^{2} / 2 \sigma^{2} \ln \delta$ and $\tau(\bar{q})=1-\alpha_{0} \bar{q}+\bar{q}^{2} \sigma^{2}(\ln \delta) / 2$, respectively. We see that $a_{3 \bar{q}}=1$.

The dependence of the parameters $\alpha_{0}$ and $\sigma$ on the intermittency exponent $\mu$ is determined with the help of the two independent equations, i.e., the energy conservation: $\left\langle\epsilon_{n} / \epsilon\right\rangle=1$ (equivalently, $\tau(1)=0$ ), and the definition of the intermittency exponent $\mu:\left\langle\epsilon_{n}^{2} / \epsilon^{2}\right\rangle=\delta_{n}^{-\mu}$ (equivalently, $\mu=1+\tau(2)$ ). Here, $\epsilon$ is the energy input rate to the largest eddies. The parameters are specified by means of $\mu$ as $\alpha_{0}=1+\mu / 2$ and $\sigma^{2}=\mu / \ln \delta$. Then, we have $f(\alpha)=1-\left(\alpha-\alpha_{0}\right)^{2} / 2 \mu$ and $\tau(\bar{q})=(1-\bar{q}) D_{\bar{q}}$ with the generalized dimension $D_{\bar{q}}=1-\mu \bar{q} / 2$, which are the same as derived in [15]. We know that $\alpha_{\bar{q}}=$ $\alpha_{0}-\mu \bar{q}$.

The explicit form of the PDF (1) for $\xi_{n}^{*} \leq\left|\xi_{n}\right|<\infty$ is found to be

$$
\hat{\Pi}_{\phi}^{(n)}\left(\xi_{n}\right)=\bar{\Pi}_{\phi}^{(n)} \frac{\bar{\xi}_{n}}{\left|\xi_{n}\right|} \exp \left[-\frac{\left(3 \ln \left|\xi_{n} / \xi_{n, 0}\right|\right)^{2}}{2 \phi^{2} \mu\left|\ln \delta_{n}\right|}\right] .
$$

The connection point is given by $\alpha^{*}=\alpha_{0}-(\sqrt{3}-1) \phi \mu / 3$.

\section{$4 \quad P$ model}

The distribution function $P^{(n)}(\alpha)$ for the $p$ model [14,15] is specified based on the binomial multiplicative process in the form [1] $P^{(n)}(\alpha)=\left(Z_{0}^{(n)}\right)^{-1}\left[2 y^{y}(1-\right.$ $\left.y)^{1-y}\right]^{-n}$ with $y=y(\alpha)=\left[\alpha+\log _{2}(1-p)\right] / \log _{2}[(1-p) / p]$ and the partition 
function $Z_{0}^{(n)}=\sqrt{\pi / 2 n} \log _{2}[(1-p) / p]$ for $n \gg 1$. The multifractal spectrum is given by $f(\alpha)=-\left\{y(\alpha) \log _{2} y(\alpha)+[1-y(\alpha)] \log _{2}[1-y(\alpha)]\right\}$, which leads to the mass exponent $\tau(\bar{q})=\log _{2}\left[p^{\bar{q}}+(1-p)^{\bar{q}}\right]$. We have $f^{\prime \prime}\left(\alpha_{\bar{q}}\right)=-\ln 2 /\{\ln [(1-$ $p) / p]\}^{2} y_{\bar{q}}\left(1-y_{\bar{q}}\right)$ with $y_{\bar{q}}=y\left(\alpha_{\bar{q}}\right)=p^{\bar{q}} /\left[p^{\bar{q}}+(1-p)^{\bar{q}}\right], \alpha_{\bar{q}}=-\left[p^{\bar{q}} \log _{2} p+(1-\right.$ $\left.p)^{\bar{q}} \log _{2}(1-p)\right] /\left[p^{\bar{q}}+(1-p)^{\bar{q}}\right]$, and then we obtain $a_{3 \bar{q}}=2\left[p^{\bar{q}}(1-p)^{\bar{q}}\right]^{1 / 2} /\left[p^{\bar{q}}+\right.$ $\left.(1-p)^{\bar{q}}\right]$. We see that, for $p>1 / 2, \alpha_{\min }=-\log _{2} p, \alpha_{\max }=-\log _{2}(1-p)$.

The dependence of $p$ on the intermittency coefficient $\mu$ is derived through its definition $\mu=1+\tau(2)$ to give $p=\left(1+\sqrt{2^{\mu}-1}\right) / 2$.

The explicit form of the $\operatorname{PDF}(1)$ for $\xi_{n}^{*} \leq\left|\xi_{n}\right| \leq \xi_{n}^{\max }$ reduces to

$$
\hat{\Pi}_{\phi}^{(n)}\left(\xi_{n}\right)=\bar{\Pi}_{\phi}^{(n)} \frac{\bar{\xi}_{n}}{\left|\xi_{n}\right|}\left\{\left[1+2\left(y-y_{0}\right)\right]^{1+2\left(y-y_{0}\right)}\left[1-2\left(y-y_{0}\right)\right]^{1-2\left(y-y_{0}\right)}\right\}^{-n / 2}
$$

with $y-y_{0}=3 \ln \left(\left|\xi_{n}\right| /\left|\xi_{n, 0}\right|\right) / \phi\left\{\log _{2}[(1-p) / p]\right\}\left|\ln \delta_{n}\right|$.

\section{Harmonious representation}

In the harmonious representation of MFA, we adopt the Tsallis-type distribution function $[2,3] P^{(n)}(\alpha)=\left(Z_{0}^{(n)}\right)^{-1}\left\{1-\left[\left(\alpha-\alpha_{0}\right) / \Delta \alpha\right]^{2}\right\}^{n /(1-q)}$ with $(\Delta \alpha)^{2}=2 X /[(1-q) \ln 2]$ and the appropriate partition function $Z_{0}^{(n)}$. Here, $q$ is the entropy index introduced in the definitions of the Rényi [17] and the Tsallis entropies [16,18]. This distribution function provides us with the multifractal spectrum $f(\alpha)=1+(1-q)^{-1} \log _{2}\left[1-\left(\alpha-\alpha_{0}\right)^{2} /(\Delta \alpha)^{2}\right]$ which, then, produces the mass exponent $\tau(\bar{q})=1-\alpha_{0} \bar{q}+2 X \bar{q}^{2}\left(1+C_{\bar{q}}^{1 / 2}\right)^{-1}+(1-q)^{-1}\left[1-\log _{2}(1+\right.$ $\left.\left.C_{\bar{q}}^{1 / 2}\right)\right]$ with $C_{\bar{q}}=1+2 \bar{q}^{2}(1-q) X \ln 2$. We have $f^{\prime \prime}\left(\alpha_{\bar{q}}\right)=-C_{\bar{q}}^{1 / 2}\left(1+C_{\bar{q}}^{1 / 2}\right) / 2 X$ with $\alpha_{\bar{q}}=\alpha_{0}-2 \bar{q} X /\left(1+C_{\bar{q}}^{1 / 2}\right)$, hence $a_{3 \bar{q}}=\left\{2 /\left[C_{\bar{q}}^{1 / 2}\left(1+C_{\bar{q}}^{1 / 2}\right)\right]\right\}^{1 / 2}$. We see that $\alpha_{\min / \max }=\alpha_{0} \mp \Delta \alpha$.

The dependence of the parameters $\alpha_{0}, X$ and $q$ on the intermittency exponent $\mu$ is determined, self-consistently, with the help of the three independent equations, i.e., the energy conservation: $\tau(1)=0$, the definition of the intermittency exponent $\mu: \mu=1+\tau(2)$, and the scaling relation:[30] $1 /(1-q)=$ $1 / \alpha_{-}-1 / \alpha_{+}$with $\alpha_{ \pm}$satisfying $f\left(\alpha_{ \pm}\right)=0$. Within the range $0.13 \leq \mu \leq 0.40$ where most of the experiments are covered, the self-consistent equations are solved, numerically, to give [6] $\alpha_{0}=0.9989+0.5814 \mu, X=1.198 \mu$ and $q=-1.507+20.58 \mu-97.11 \mu^{2}+260.4 \mu^{3}-365.4 \mu^{4}+208.3 \mu^{5}$. It is interesting to compare the first two with the corresponding equations for the log-normal model $(q=1)$, i.e., $\alpha_{0}=1+0.5 \mu$ and $X=\mu$. 

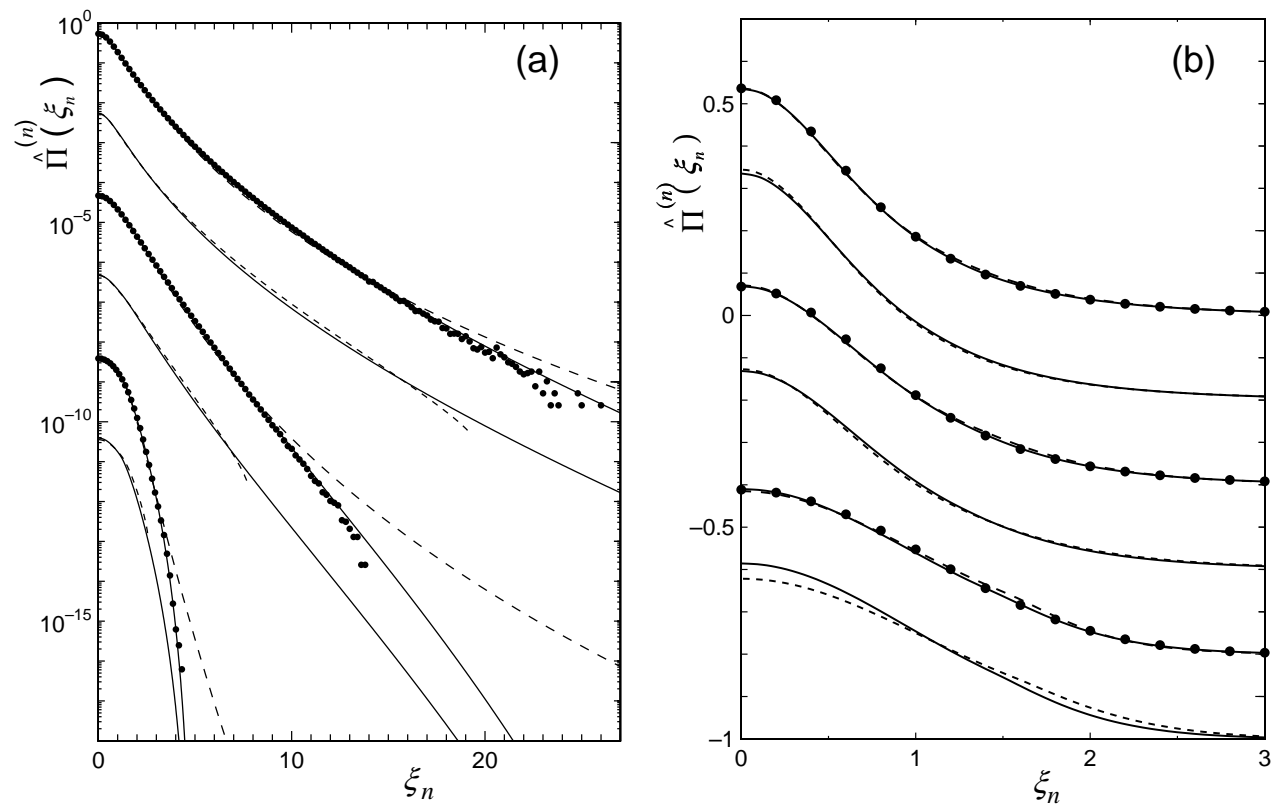

Fig. 1. Analyses of the PDF's of the velocity fluctuations (closed circles) for three different measuring distances, observed by Gotoh et al. at $R_{\lambda}=380$, with the help of the PDF's $\hat{\Pi}^{(n)}\left(\xi_{n}\right)$ by the harmonious representation (solid line) and by the log-normal model (dashed line) are plotted on (a) log and (b) linear scales. The PDF's by the $p$ model (dotted line) are compared with the PDF's by the harmonious representation (solid line). Comparisons are displayed in pairs. The solid lines in each set of pairs are the same. For better visibility, each PDF is shifted by -2 unit in (a) and by -0.2 in (b) along the vertical axis. Parameters are given in the text.

The explicit form of the $\operatorname{PDF}(1)$ for $\xi_{n}^{*} \leq\left|\xi_{n}\right| \leq \xi_{n}^{\max }$ turns out to be

$$
\hat{\Pi}_{\phi}^{(n)}\left(\xi_{n}\right)=\bar{\Pi}_{\phi}^{(n)} \frac{\bar{\xi}_{n}}{\left|\xi_{n}\right|}\left[1-\frac{1-q}{n} \frac{\left(3 \ln \left|\xi_{n} / \xi_{n, 0}\right|\right)^{2}}{2 \phi^{2} X\left|\ln \delta_{n}\right|}\right]^{n /(1-q)} .
$$

\section{Analysis of experiments}

The scaling exponents $\zeta_{m}$ of velocity structure function reported by Gotoh et al. [19] are analyzed by the method of the least squares (MLS) with the theoretical formulae of the harmonious representation, of the log-normal model and of the $p$ model, giving, respectively, the values of the intermittency exponent $\mu=0.240,0.217$ and 0.249 .

In Fig. 1, the PDF's of the velocity fluctuations (closed circles) measured by Gotoh et al. in their DNS at $R_{\lambda}=380$ [19] for three different measuring distances, $r / \eta=\ell_{n} / \eta=2.38,19.0,1220$ from the top set of pairs to the bottom set, are analyzed with the help of the PDF's $\hat{\Pi}^{(n)}\left(\xi_{n}\right)$ of the harmonious representation (solid line) and of the log-normal model (dashed line). Here, 

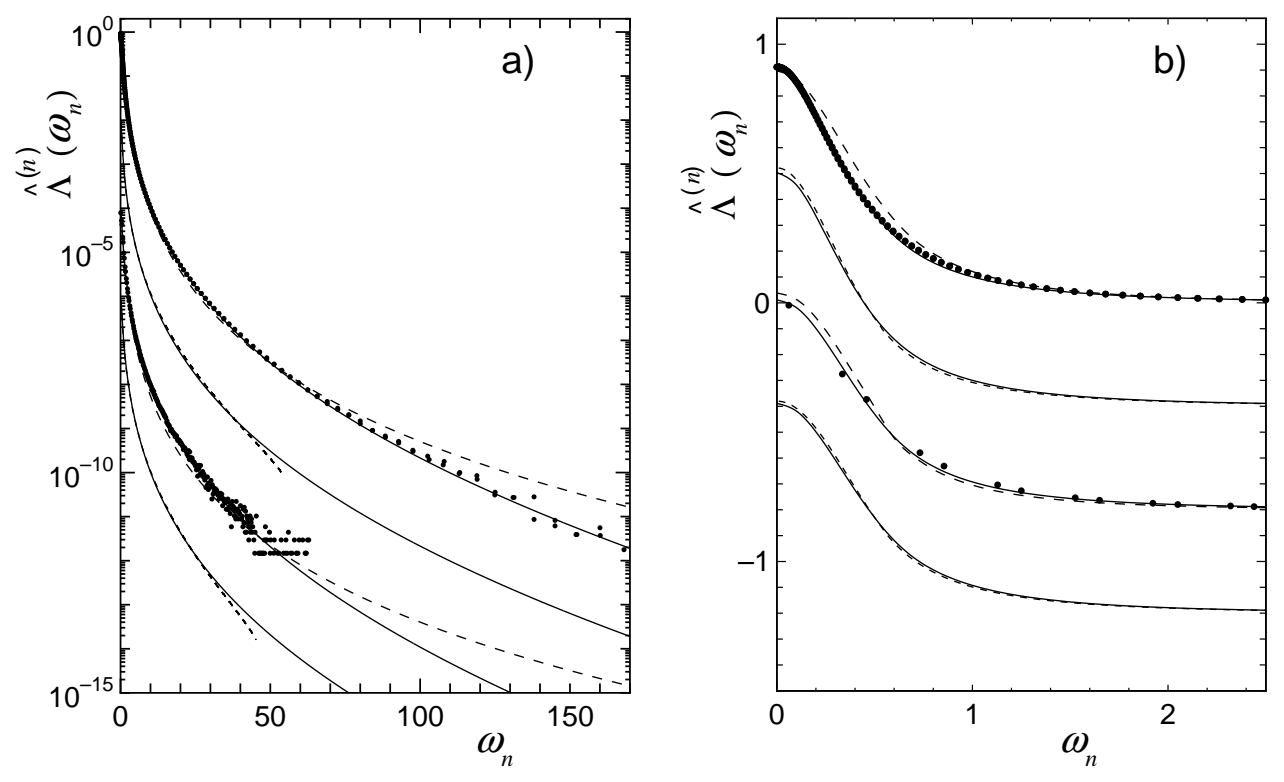

Fig. 2. Analyses of the PDF's of fluid particle accelerations, measured by Gotoh et al. at $R_{\lambda}=380$ (circles in the top set) and by Bodenschatz et al. at $R_{\lambda}=690$ (circled in the bottom set), by means of the PDF's $\hat{\Lambda}^{(n)}\left(\omega_{n}\right)$ by the harmonious representation (solid line) and by the log-normal model (dashed line) are plotted on (a) log and (b) linear scales. The PDF's by the $p$ model (dotted line) are compared with the PDF's by the harmonious representation (solid line). Results are displayed in pairs. The solid lines in each set of pairs are the same. For better visibility, each PDF is shifted by -2 unit in (a) and by -0.4 in (b) along the vertical axis. Parameters are given in the text.

$\eta=\left(\nu^{3} / \epsilon\right)^{1 / 4}$ represents the Kolmogorov scale. The DNS data points are symmetrized by taking averages of the left and the right hand sides data. In each set, a comparison of the PDF of the harmonious representation (solid line) with the PDF of the $p$ model (dotted line) is given. For the harmonious PDF's (solid line), $q=0.391$ ( $\mu=0.240)$, and, from the top set to the bottom set, $\left(n, q^{\prime}\right)=(20.7,1.60),(13.6,1.50),(6.10,1.20), \xi_{n}^{*}=1.10,1.23,1.43\left(\alpha^{*}=\right.$ $1.07)$ and $\xi_{n}^{\max }=204,38.2,6.63$. For the PDF by the log-normal model (dashed line), from the top set to the bottom set, $\left(n, q^{\prime}\right)=(21.5,1.70),(13.0,1.63)$, $(5.00,1.24)$ and $\xi_{n}^{*}=1.19,1.34,1.51\left(\alpha^{*}=1.06\right)$. For the PDF by the $p$ model (dashed line), from the top set to the bottom set, $\left(n, q^{\prime}\right)=(21.0$, $1.60),(13.0,1.62),(5.50,1.20), \xi_{n}^{*}=1.06,1.07,1.55\left(\alpha^{*}=1.08\right)$ and $\xi_{n}^{\max }=$ $19.3,7.70,3.31$. The tail of PDF of the $p$ model stops at $\xi_{n}^{\max }$ which is smaller than the maximum value of measured data point for each measuring distance. Note that every PDF's are plotted on (a) log and (b) linear scales.

In Fig. 2, the PDF's of the fluid particle accelerations (closed circles) reported by Gotoh et al. at $R_{\lambda}=380$ (top set) [20] and by Bodenschatz et al. at $R_{\lambda}=690$ (bottom set) $[21,22,23]$ are analyzed with the PDF's $\hat{\Lambda}^{(n)}\left(\omega_{n}\right)$ of the harmonious representation (solid line) and of the log-normal model (dashed line). The measured data points both on the left and right hand sides of the 
PDF's are shown altogether on one side by closed circles in the figure. In each set, a comparison of the PDF of the harmonious representation (solid line) with the PDF of the $p$ model (dotted line) is given. For the harmonious PDF's (solid line), from the top set of pairs to the bottom set, $q=0.391,0.391$ $(\mu=0.240,0.240),\left(n, q^{\prime}\right)=(17.5,1.70),(17.1,1.45) \omega_{n}^{*}=0.622,0.605\left(\alpha^{*}=\right.$ $1.01,1.01)$, and $\omega_{n}^{\max }=2530,2040$. For the PDF by the log-normal model (dashed line), from the top set to the bottom set, $\left(n, q^{\prime}\right)=(17.0,1.50),(18.5$, $1.04), \omega_{n}^{*}=0.644,0.558\left(\alpha^{*}=1.00,1.00\right)$, and $\omega_{n}^{\max }=49.6,76.6$. For the PDF by the $p$ model (dotted line), from the top set to the bottom set, $\left(n, q^{\prime}\right)=$ $(19.0,1.50),(18.5,1.20) \omega_{n}^{*}=0.539,0.547\left(\alpha^{*}=1.01,1.01\right)$, and $\omega_{n}^{\max }=54.1$, 45.2. Note that every PDF's are plotted on (a) log and (b) linear scales.

The values of $n$ for all the PDF's are determined by MLS by adjusting the integrand $\xi_{n}^{4} \Pi_{\phi}^{(n)}\left(\xi_{n}\right)$ of the fourth moment both of data and of the theories. The values of $q^{\prime}$ for all the PDF's are obtained by MLS by fitting the theoretical PDF's at the center part, $\left|\xi_{n}\right| \leq \xi_{n}^{*}$, with the observed PDF's.

\section{Discussions}

As was shown in this paper, the harmonious representation within MFA provides the highest accuracy in analyzing observed PDF's. This high accuracy allows us to extract some information about the dynamics underlying turbulence. For example, through the analysis of the velocity fluctuations in Fig. 1, we extracted the dependence of $q^{\prime}$ on $r / \eta$ as [9] $q^{\prime}=1.71-0.05 \log _{2}(r / \eta)$. We then obtain the diffusion coefficient for the fluctuations of magnitude $\left|\xi_{n}\right|\left(\leq \xi_{n}^{*}\right)$ in the form $D_{\phi}\left(\xi_{n}\right) / D_{\phi}(0)=1+\left[0.71-0.05 \log _{2}(r / \eta)\right][(\phi+$ $\left.\left.3 f^{\prime}\left(\alpha^{*}\right)\right) / 2 \phi\right]\left(\xi_{n} / \xi_{n}^{*}\right)^{2}$. Note that this is dependent on the distance $r$ of two measuring points. In deriving this, we assumed that the drift term is linear since the magnitude of the fluctuations in the center part of the PDF is smaller than the order of their standard deviations $\left(\left|\xi_{n}\right| \leq 1\right)$, and, therefore, used the formula for anomalous diffusion given in [29]. The investigation to this direction is in progress, and will be reported elsewhere.

\section{Acknowledgements}

The authors would like to thank Prof. C. Tsallis for his continuous encouragement, and Dr. A.M. Reynolds for the fruitful discussions with him. The authors are grateful to Prof. E. Bodenschatz and Prof. T. Gotoh for the kindness to show their data prior to publication. 


\section{References}

[1] T. Arimitsu and N. Arimitsu, Phys. Rev. E 61 (2000) 3237.

[2] T. Arimitsu and N. Arimitsu, J. Phys. A: Math. Gen. 33 (2000) L235 [CORRIGENDUM: 34 (2001) 673].

[3] T. Arimitsu and N. Arimitsu, Physica A 295 (2001) 177.

[4] N. Arimitsu and T. Arimitsu, J. Korean Phys. Soc. 40 (2001) 1032.

[5] T. Arimitsu and N. Arimitsu, J. Phys.: Condens. Matter 14 (2002) 2237.

[6] N. Arimitsu and T. Arimitsu, Europhys. Lett. 60 (2002) 60.

[7] T. Arimitsu and N. Arimitsu, Cond. Matter Phys. 6 (2003) 85.

[8] T. Arimitsu and N. Arimitsu, (2002) cond-mat/0210274.

[9] T. Arimitsu and N. Arimitsu, In Highlights in Condensed Matter Physics, editors, C. Noce A. Avella, R. Citro and M. Salerno (AIP Conference Proceedings 695, 2003) in press.

[10] A.N. Kolmogorov, C.R. Acad. Sci. USSR 30 (1941) 301; 538.

[11] A.M. Oboukhov, J. Fluid Mech. 13 (1962) 77.

[12] A.N. Kolmogorov, J. Fluid Mech. 13 (1962) 82.

[13] A.M. Yaglom, Sov. Phys. Dokl. 11 (1966) 26.

[14] C. Meneveau and K. R. Sreenivasan, Phys. Rev. Lett. 59 (1987) 1424.

[15] C. Meneveau and K. R. Sreenivasan, Nucl. Phys. (Proc. Suppl.) B 2 (1987) 49.

[16] C. Tsallis, J. Stat. Phys. 52 (1988) 479.

[17] A. Rényi, Proc. 4th Berkeley Symp. Maths. Stat. Prob. 1 (1961) 547.

[18] J.H. Havrda and F. Charvat, Kybernatica 3 (1967) 30.

[19] T. Gotoh, D. Fukayama and T. Nakano, Phys. Fluids 14 (2002) 1065.

[20] T. Gotoh, private communication

[21] A. La Porta, G. A. Voth, A. M. Crawford, J. Alexander and E. Bodenschatz Nature 409 (2001) 1017.

[22] G. A. Voth, A. La Porta, A. M. Crawford, J. Alexander and E. Bodenschatz, J. Fluid Mech. 469 (2001) 121.

[23] A. M. Crawford, N. Mordant and E. Bodenschatz, (2002) physics/0212080

[24] R. Benzi, G. Paladin, G. Parisi and A. Vulpiani, J. Phys. A: Math. Gen. 17 (1984) 3521. 
[25] S. S. Moiseev, A. V. Tur and V. V. Yanovskii, Sov. Phys. JETP 44 (1976) 556.

[26] U. Frisch and G. Parisi, in Turbulence and Predictability in Geophysical Fluid Dynamics and Climate Dynamics, ed. by M. Ghil, R. Benzi and G. Parisi (North-Holland, New York, 1985) 84.

[27] U.M.S. Costa, M.L. Lyra, A.R. Plastino and C. Tsallis, Phys. Rev. E 56 (1997) 245.

[28] M.L. Lyra and C. Tsallis, Phys. Rev. Lett. 80 (1998) 53.

[29] G. Kaniadakis and G. Lapenta, Phys. Rev. E 62 (2000) 3246.

[30] The scaling relation is a generalization of the one derived first in $[27,28]$ to the case where the multifractal spectrum has negative values [2]. 